\title{
Highlights from the latest articles in nano-oncology
}

\author{
Shreya Goel ${ }^{1}$, Feng Chen ${ }^{2}$, and Weibo Cai $^{*}, 2,3,4$ \\ ${ }^{1}$ Materials Science Program, University of Wisconsin-Madison, WI, USA \\ ${ }^{2}$ Department of Radiology, University of Wisconsin-Madison, WI, USA \\ ${ }^{3}$ Department of Medical Physics, University of Wisconsin-Madison, WI, USA \\ ${ }^{4}$ University of Wisconsin Carbone Cancer Center, Madison, WI, USA
}

\begin{abstract}
Green tea-based drug carrier for synergistically enhanced therapeutic effects
Evaluation of: Chung JE, Tan S, Gao SJ et al. Self-assembled micellar nano-complexes comprising green tea catechin derivatives and protein drugs for cancer therapy. Nat. Nanotechnol. 9(11), 907-912 (2014).
\end{abstract}

Drug delivery has immensely benefited from the development of nanovehicles which aim to improve drug solubility and bioavailability, along with with optimizing the pharmacokinetics and biodistribution, among others. Decades of research has focused on developing more biocompatible and non-toxic nanocarriers. However, the toxicity generated from metabolism and retention of the excipients remains a major cause of concern. In this study, Chung and coworkers have improved upon the design of a micellar drug-delivery system where the carrier itself displays therapeutic effects. The authors have utilized a green tea component, (-)-epigallocatechin-3-O-gallate (EGCG; which possesses anticancer effects) and its derivatives, oligomerized EGCG (OEGCG) and PEG-EGCG, to encapsulate Herceptin (a humanized anti-HER2 monoclonal antibody that has been approved to treat meta-static breast cancer) to create self-assembled, monodispersed and spherical micellar nano-complexes (MNC), denoted as Herceptin-MNC; with a hydrodynamic diameter of $\sim 90$ $\mathrm{nm}$. The PEG-EGCG shell around the Herceptin-OEGCG core ensured reduced immunogenicity and prevented proteolysis and functional loss of Herceptin. When tested in vivo and in vitro, Herceptin-MNCs displayed synergistically enhanced therapeutic effects both in BT-474 breast cancer cell lines and tumor xenografts. Moreover, the nanocomplexes displayed better tumor specificity and longer blood half-life than Herceptin alone. Overall, this study developed a smart drug-delivery system that exhibited combinatorial therapeutic

\footnotetext{
(C) 2015 Future Medicine Ltd

*Author for correspondence: wcai@uwhealth.org.

For reprint orders, please contact: reprints@ futuremedicine.com

Financial \& competing interests disclosure

This work is supported, in part, by the University of Wiscon-sin-Madison, the National Institutes of Health (NIBIB/NCI 1R01CA169365 and P30CA014520), the Department of Defense (W81XWH-11-1-0644) and the American Cancer Society (125246RSG-13-099-01-CCE). The authors have no other relevant affiliations or financial involvement with any organization or entity with a financial interest in or financial conflict with the subject matter or materials discussed in the manuscript apart from those disclosed. No writing assistance was utilized in the production of this manuscript.
} 
effects between the drug and the excipient and overcame the toxicity concerns at the same time.

Organic-inorganic hybridization strategy for bioresponsive and tunable organosilica nanoplatforms Evaluation of: Chen Y, Meng Q, Wu M et al. Hollow mesoporous organosilica nanoparticles: a generic intelligent framework-hybridization approach for biomedicine. J. Am. Chem. Soc. 136(46), 16326-16334 (2014).

Inorganic mesoporous silica nanoparticles (MSNs) have faced many challenges to clinical translation, despite their promising results in drug/gene delivery and imaging/therapy studies in animal tumor models. A major concern arises from the biosafety of the inert -Si-O-Sibackbone. In this paper, Chen et al. have proposed an effective way to circumvent this issue by incorporating physiologically active thioether groups into the framework of MSNs. The authors report the synthesis of 400 -nm-sized hybrid hollow mesoporous organosilica nanoparticles (HMONs) with up to quintuple molecularly organic-inorganic hybridizations using different bissilylated precursors. Extensive characterizations demonstrated welldefined and uniform mesoporous skeleton ( $3.8 \mathrm{~nm}$ pore size), high surface area $\left(1005 \mathrm{~m}^{2} / \mathrm{g}\right)$ and high pore volumes $\left(\sim 1.09 \mathrm{~cm}^{3} / \mathrm{g}\right)$, which are indicative of high drug loading capacity. Unique high intensity ultrasonic frequency responsive drug releasing behavior and enhanced ultrasonographic effects were observed in doxorubicin (Dox)-loaded HMONs. In addition, Dox loaded HMONs demonstrated significantly higher (approximately twofold) tumoricidal effects than free Dox, as well as substantially better inhibition of tumor metastasis to the lungs. In conclusion, the authors successfully incorporated the advantages from both silica nanomaterials (high stability, easy tunability and facile functionalization) and organic carriers (higher biocompatibility and biodegradability) into one simple, tunable and versatile nanoplatform with enhanced therapeutic effects and clinical potential.

Inorganic nanoparticles for first-in-human molecular imaging of cancer Evaluation of: Phillips E, Penate-Medina O, Zanzonico PB et al. Clinical translation of an ultrasmall inorganic optical-PET imaging nanoparticle probe. Sci. Transl. Med. 6(260), 260ra149 (2014).

This study marks a major milestone in the field of nanooncology, particularly for inorganic nanoprobes designed for diagnosis and imaging applications. Phillips and coworkers report a first-in-human trial of ultrasmall $(\sim 6 \mathrm{~nm})$ hybrid core-shell nanoparticles (Cy5 dye encapsulated in a silica shell) called Cornell dots (or C-dots), for dual-modality PET and optical imaging in metastatic melanoma patients. The nanoparticles were modified with the peptide cyclo-(Arg-Gly-Asp-Tyr) (cRGDY) for integrin-mediated tumor targeting, and labeled with ${ }^{124}$ I for PET imaging. Single-dose administration of ${ }^{124}$ I-cRGDY-PEG-C dots demonstrated dominant renal clearance with limited uptake in the reticuloendothelial system, indicating good safety profile over a 2 -week period. Accumulation of ${ }^{124}$ I-cRGDYPEG-C dots at tumor sites in some patients in this study is expected to assist in future cancer screening and staging, patient selection for integrin targeted therapy and possibly imageguided surgery. While the present trial proves valuable in estimating nanoparticle dose requirements, future studies will be needed in larger cohorts of patients, to evaluate nonspecific localization of $\mathrm{C}$-dots due to enhanced permeability and retention effects in the 
tumor tissue, as well as functionalization with other targeting ligands tailored for personalized cancer imaging. Overall, this study realizes the much awaited translation of inorganic, cancer-targeted nanoparticles from bench to bedside. Although there will be many years before such nanoparticle-based imaging agents can be approved by the US FDA for routine clinical diagnosis, the favorable pharmacokinetics, complete renal excretion and minimal off-target toxicity observed in the current study will greatly encourage future translational research of other nanoparticles into clinical investigation.

\section{Engineering of platelet-like nanoparticles for enhanced vascular targeting and hemostasis Evaluation of: Anselmo AC, Modery-Pawlowski CL, Menegatti S et al.} Platelet-like nanoparticles: mimicking shape, flexibility, and surface biology of platelets to target vascular injuries. ACS Nano. 8(11), 11243-11253 (2014).

Given the tremendous potential of nanomaterials in the treatment of vascular disorders like cancer, inflammation and hemorrhage, among others, several strategies have been employed to home them specifically to the sites of vascular injury. However, owing to the vastly different characteristics from the circulating cells in the blood, traditional nanomaterials perform suboptimally in this regard. Anselmo et al. report the synthesis of novel platelet-like nanoparticles (PLNs) inspired by the superior circulation, targeting, vascular margination and site-specific adhesion properties of natural platelets, which are highly desired features of synthetic nanoparticles. PLNs ( $200 \mathrm{~nm})$ synthesized via layer-by-layer assembly, upon functionalization with wound and platelet specific peptides, mimicked the discoid shape, mechanical flexibility and complex biochemical interactions of platelets. Rigorous biophysical and biochemical characterizations revealed that flexible discoidal PLNs are better than rigid spherical PLNs. Moreover, enhanced site-specific accumulation and surface adhesion were observed for the former both in vitro and in vivo. About $65 \%$ reduction in bleeding time was achieved in mouse tail transection models for flexible discoidal PLNs, indicating improved hemostatic functions. Future studies to determine dose-dependent impact on physiological and immunogenic functions in vivo have also been proposed. This study is of high significance in developing next-generation, bioinspired nanoplatforms which can serve not only as hemostats in wound healing, but also as targeted therapeutic devices for vascular diseases like atherosclerosis and restenosis. 